\title{
Analysis on Influence Model of Network Education Based on SPSS and Test Technology
}

\author{
Junling $\mathrm{Wei}^{1}$, Mingxia Zhu ${ }^{2}$ \\ Polytechnic College, ${ }^{1}$ Hebei University of science and Technology, Shijiazhuang, China \\ junlingwei@126.com \\ ${ }^{2}$ School of Environmental Science and Engineering, Hebei University of science and Technology \\ Shijiazhuang, China
}

Keywords:network; youth moral quality; contrast matrix; weight vector; consistency check

\begin{abstract}
With the development of Internet technology, the network environments become more and more complicated, which filled with unhealthy web information and vulgar network games. In China, young people are the main groups contacting with network. Youth moral character is susceptible to the network environment. This article studies the influence of network to our youth moral character development. First of all, this paper uses multilevel evaluation theory and introduces contrast matrix and consistency check principle of weight vector and takes the network pornography, violence, ethics, terror and drugs and so on as the main contents. It establishes the evaluation model based on the influence of network on youth moral quality. It uses the SPSS statistical analysis software to analyze the assessment information and gets the influence degree of different age, different regions and different gender on youth moral character development. At last, it puts forward youth network moral quality education countermeasures and provides the theory reference for the development youth moral quality.
\end{abstract}

\section{Introduction}

Young people are the main group to contact with network. How young people to grasp the moral quality measurement and make their own moral standard better is the focus of this paper with the increasingly complicated network environment[1-3]. This paper first introduces the multi-level model of the development of youth moral quality. It gives out the schematic diagram and structures comparison matrix and stratified weight coefficient through the mathematical formula[4,5]. At last, it analyzes the score data combined with SPSS software[6,7]. It puts forward the network moral education countermeasures for results of the analysis and provides the reliable theory basis for young people network moral development.

\section{The multilevel model of influences of network on youth moral quality}

Analytic hierarchy process (AHP) is process data hierarchically which general divided into three layers: target layer, principle layer and subset principle layer. These three layers directly construct comparison matrix[8,9]. It establishes direct contact weight through the weight of each layer. It grasps the layers until gets the relevant data of the last layer. This paper marks the youth moral development level uses grade evaluation. The specific marking standard is shown in Table 1.

TABLE I. THE EVALUATION TABLE OF MORAL EFFECT ON NETWORK
\begin{tabular}{|c|l|l|l|l|}
\hline Index/score & 0 & 10 & 20 & 30 \\
\hline Effect of pornography & No & Slight & The heavier & Serious \\
\hline Effects of violence & No & Slight & The heavier & Serious \\
\hline Horror effects & No & Slight & The heavier & Serious \\
\hline Ethical implications & No & Slight & The heavier & Serious \\
\hline The influence of drugs & No & Slight & The heavier & Serious \\
\hline
\end{tabular}

From Table 1 we can see that the influence of network on youth moral development level mainly contains network pornography, violence, ethics, terror and drugs and so on. Each of them can be divided into four grades. The slightest is zero,and the highest and serious influence is thirty. 


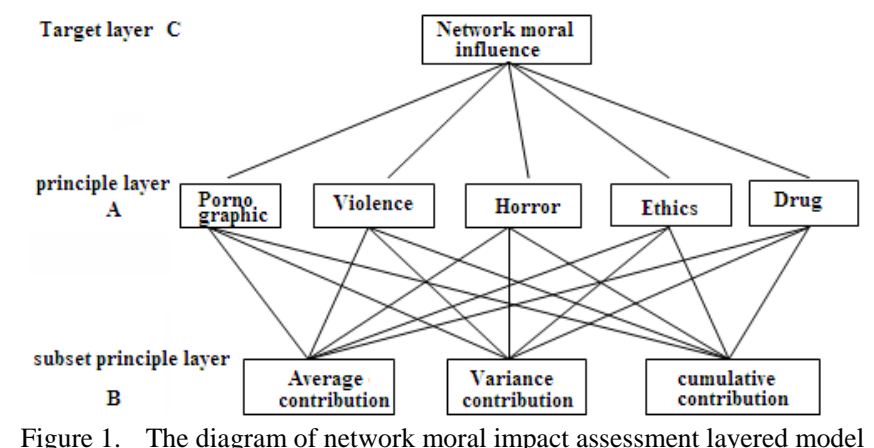

Figure 1 is the diagram of network moral impact assessment layered model. From the chart we can see that the evaluation of youth moral quality impact of the network can be divided into three layers. The target layer is influenced by network moral. Principle layer and subset principle layer need to build weight coefficient contact ion which need to confirm the contrast matrix B.

We first study the nature of the consistent matrix to confirm the weight vector of target $\mathrm{C}$ to A1, $\mathrm{A} 2 \ldots$ An by $\mathrm{B}$. We can call the positive reciprocal matrix of $b_{i j} \cdot b_{j l}=b_{i l}, i, j, l=1,2, \cdots, n$ for consistent matrix. For example[10]:

$$
B=\left(\begin{array}{cccc}
\frac{m_{1}}{m_{1}} & \frac{m_{1}}{m_{2}} & \cdots & \frac{m_{1}}{m_{n}} \\
\frac{m_{2}}{m_{1}} & \frac{m_{2}}{m_{2}} & \cdots & \frac{m_{2}}{m_{n}} \\
\vdots & \vdots & \ddots & \vdots \\
\frac{m_{n}}{m_{1}} & \frac{m_{n}}{m_{2}} & \cdots & \frac{m_{n}}{m_{n}}
\end{array}\right) \quad \quad \mathrm{B}=\left(\begin{array}{ccccc}
2 & \frac{1}{3} & 1 & 4 & 2 \\
3 & 7 & 2 & 5 & 3 \\
\frac{1}{2} & \frac{1}{4} & 1 & \frac{1}{7} & \frac{1}{5} \\
\frac{1}{4} & \frac{1}{8} & 5 & 3 & 2 \\
\frac{1}{3} & \frac{1}{5} & 3 & 1 & 1
\end{array}\right)
$$

The rank of matrix B is 1.n is the only nonzero characteristic root of B.

According to the Table 1 we can structure the contrast matrix:

Multilayer evaluation model need to carry out the consistency check. Consistency index is[11]:

$$
\mathrm{S}=\frac{\lambda-k}{k-1}
$$

$S$ is bigger, the inconsistency is more serious. The consistency index can be calculated as:

$$
C I=\frac{4.028-4}{4-1}=0.0933
$$

The weight vector of principle layer to target layer is[12]:

$$
m^{(2)}=\left(m_{1}^{(2)}, \cdots, m_{n}^{(2)}\right)^{T}
$$

The weight vector of principle layer to subset principle layer is[13]:

$$
m_{v}^{(3)}=\left(m_{v 1}^{(3)}, \cdots, m_{v z}^{(3)}\right)^{T}, v=1,2, \cdots, n
$$

We can structure the matrix[14]:

$$
M^{(3)}=\left(m_{1}^{(3)}, \cdots, m_{n}^{(3)}\right)
$$

$\mathrm{M}$ is combination weight vector.

\section{The multilevel statistical analysis influences of network on youth moral quality}

This paper marks and evaluates the influence of network on youth moral through 10000 young people of different ages, different areas and different genders. It analyzes the results by using SPSS software. The statistical analysis of the general parameters is shown in Table 2. 
TABLE II. ANALYSIS OF SPSS STATISTICAL MORAL EVALUATION CONTRIBUTION

\begin{tabular}{|c|l|l|l|}
\hline Essential factor & \multicolumn{1}{|c|}{$\begin{array}{c}\text { Variance contribution } \\
\text { The cumulative contribution } \\
\text { rate(\%) }\end{array}$} \\
\hline Pornographic & 2.53 & 26.23 & $35.23 \%$ \\
\hline Violence & 1.18 & 25.12 & $21.95 \%$ \\
\hline Horror & 1.06 & 10.08 & $21.82 \%$ \\
\hline Ethics & 1.82 & 23.25 & $8.52 \%$ \\
\hline Drug & 1.73 & 12.58 & $12.48 \%$ \\
\hline
\end{tabular}

In Table 2,the contribution of various elements is different. From the evaluation of mean value, the contribution of moral influence on pornography is the highest and the terror's is the lowest. From the view of variance contribution, the influence contribution of violence and ethical moral is also very high. So at last, we make the final evaluation using the cumulative contribution.

In order to study the influence of the network on youth moral development of different areas, we study the young people of different age from 22 to 30. In Figure 2 the results show that the effect on Shandong area is larger, northeast China is lower which may be associated with regional economic development degree.

In order to study the influence of the network on youth moral development of different areas, we study the young people of different age from 22 to 30. The result is that the effect on male is higher than female which shown in Figure 4.
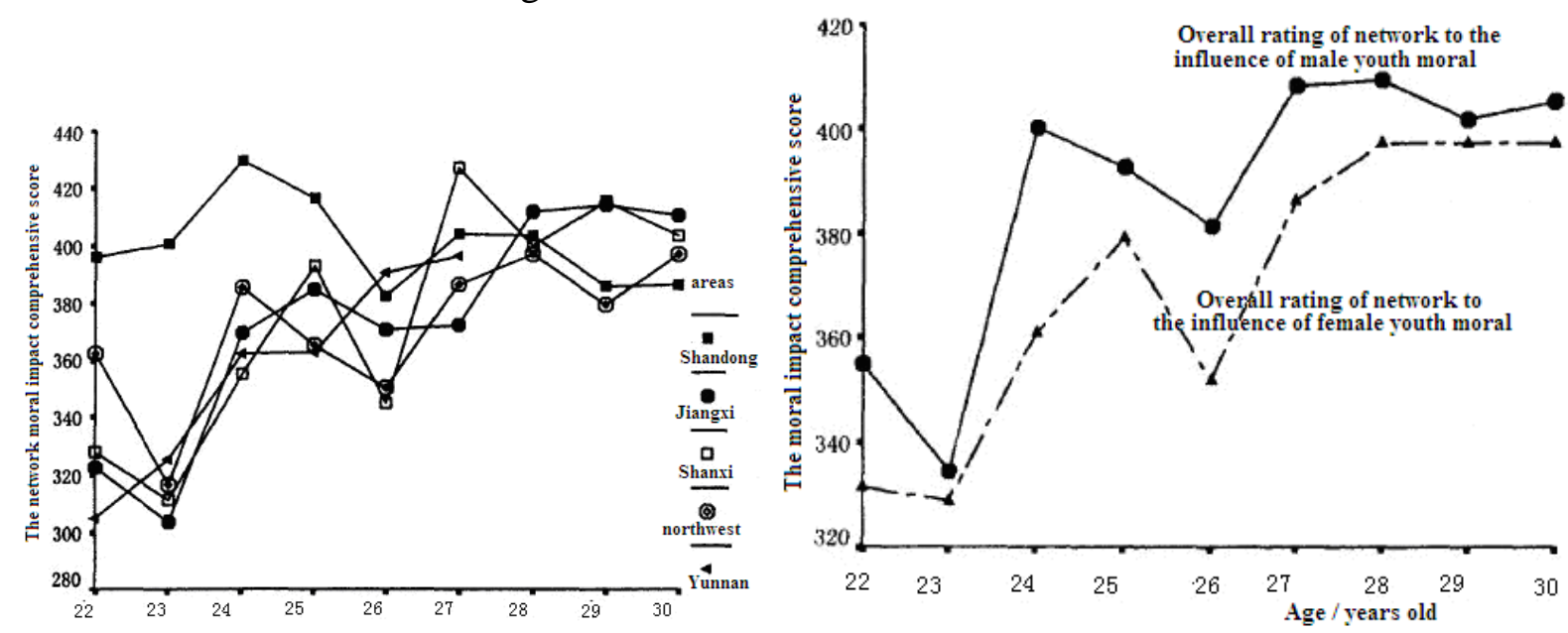

Figure 2. The influence of network on youth moral development in different areas and different ages

Figure 3. The influence network to the youth moral development of different genders and different ages

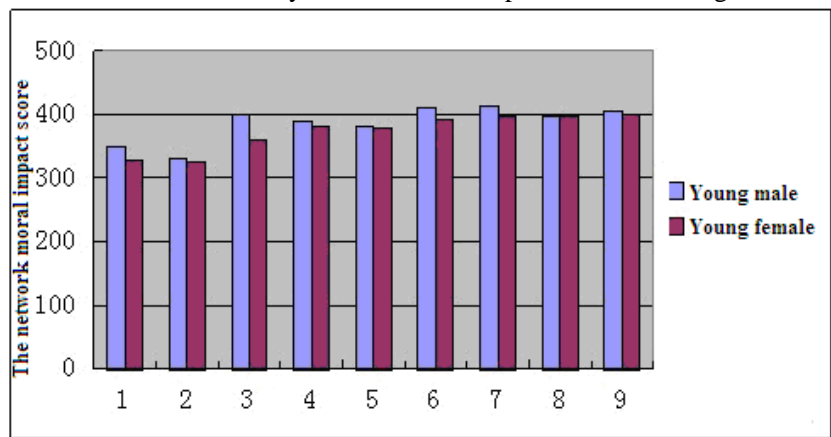

Figure 4. The columnar analysis of the effect of network on the youth moral development of different genders and different ages

\section{The countermeasures to the moral education of the youth based on network}

Through the above research data chart we can see that the network has a great influence on the development of youth moral level. It is different in different age groups, areas and genders. In order to reduce the negative effects of network on moral level of youth development, it puts forward the countermeasures which are shown as follows[15]:

(1) We can join the moral education and cultural connotation into the games

Network game is the main carrier of network and young. If the network game filled with violence and terror, it will have a significant impact on youth moral character development, especially the 
young people addicted to the network. So, we should join moral education, cultural connotation, five thousand years civilization and moral ethics into network games in order to infect and educate young people in the process of playing rather than blindly filled with vulgar taste.

(2)We should decrease the frequency of pornographic violence and terror appeared in the network

We should increase the potency dimension of regulation network and decrease the pornographic violence and terror in network videos and web sites. Youth moral quality is easy to influence. If the moral ideas of vulgar taste spread in teenagers, it will formed the bad moral atmosphere. So, the network control is the best solution.

(3) We can establish youth network moral psychological counseling centers

Young people often can not control the network moral quality. In order to develop the youth moral character to positive direction, we should open special web page and chat rooms for youth group to consult based on the network moral problems of youth.

\section{Conclusion}

This paper established the hierarchical evaluation mathematical model of influence of network moral on youth. It given the layered model diagram and established grade evaluation mechanism of the network to the youth. It established comparison matrix on the basis of hierarchical evaluation and got stratified weight coefficient. In this paper, it analyzed the statistical data using the SPSS software. In order to studied the influence of network to youth moral development in different areas and different genders, we especially selected young people of different gender from 22 to 30 . The results showed that the influence of Shandong areas is higher, the northeast is lower and influence of male youth moral development level is higher. With the increasingly complicated network environment, how to make moral level of young people who contact with network more developed towards benign direction is the focus of research. The whole society should pay attention to the young people who surfing the Internet frequently and provide a good environment of network moral for young people.

\section{References}

[1] Li Gao. Evaluation of moral. Journal of Bingtuan Education Institute.2008 (03):23-29.

[2] Xiaolin Li. How to construct the new system of moral evaluation standard? . Shanxi higher school social science of sciences report,2012. (02):23-24.

[3] Huangmei Huo, Renqian Zhao, Jing zhang. The moral evaluation model and application of online games.Journal of Beijing university of posts and telecommunications (social science edition),2008 (04):34-35.

[4] Chenhong Ge. How can we evaluate the moral? . Journal of Qilu,2011 (03):21-23.

[5] Yancai Zhao. The dialysis of basic characteristics of Chinese traditional ethics. Journal of Anhui university (philosophy and social science Edition), 2006(03):21-23.

[6] Jianjun Peng. Discussion on the moral standard.Journal of Nanhua university (social science edition),2011(12):21-23.

[7] Yazheng Liu. The moral evaluation.Theoretical discussion,2007(05):23-24.

[8] Dongping Han. Talk about the moral evaluation standards and technology . Journal of Huazhong Normal University,2012(05):23-24.

[9] Yunlong Liu. The retrospect and prospect of China's moral development in twenty century. Journal of Shanxi normal university,2012(04):21-23.

[10] Yongzhong Liu. Talk about hierarchical structure system of the moral evaluation standard . Morality and civilization ,2010(06):23-25.

[11] Congya Xia. Comprehensive grasp about moral evaluation standard.Journal of Shandong social science,2011 (02):21-23.

[12] Hehang Liu. Moral evaluation and moral evaluation standard.Journal of Henan normal university (philosophy and social science edition),2011(01):23-29.

[13] Lixia Chu. The ethics think about the development of network.Tianjin social science,2010(05):21-23.

[14] Guishan Xie. Network moral meaning people. Ethics,2010(05):21-25.

[15] Lanfen Li. Talk about the ethical issues of network times. Ethics,2011(10):29-30. 